\title{
INTERWORKING IN HETEROGENEOUS WIRELESS NetWORKS: COMPREHENSIVE FramEWORK AND FUTURE TRENDS
}

\author{
Ramon Ferrus, Oriol Sallent, AND Ramon Agusti, \\ UnIVERsitat PoliteCNICA DE CATALUNYA
}

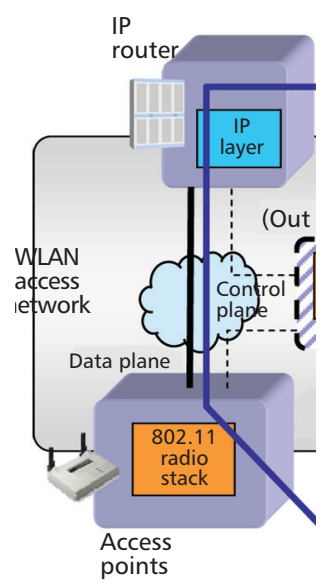

The authors develop

a comprehensive

framework to

categorize

interworking

solutions by defining

a generic set of

interworking levels

and its related key

interworking

mechanisms.

\section{ABSTRACT}

Interworking mechanisms are of prime importance to achieve ubiquitous access and seamless mobility in heterogeneous wireless networks. In this article we develop a comprehensive framework to categorize interworking solutions by defining a generic set of interworking levels and its related key interworking mechanisms. The proposed framework is used to analyze some of the most relevant interworking solutions being considered in different standardization bodies. More specifically, I-WLAN and GAN approaches for WLAN and cellular integration, solutions for WiMAX and 3GPP LTE/SAE interworking, and the forthcoming IEEE 802.21 standard are discussed from the common point of view provided by the elaborated framework.

\section{INTRODUCTION}

An intrinsic characteristic in current and future wireless communication scenarios is heterogeneity, which refers to the coexistence of multiple and diverse wireless networks with their corresponding radio access technologies (RATs). Heterogeneity is directly associated to the fact that no single RAT is able to optimally cover all the different wireless communications scenarios. Hence, a radio technology optimized to provide outdoor coverage to high mobility users may fail to meet more demanding data rates in low mobility indoor scenarios and vice versa. Heterogeneity is also inherent to technological evolution since many new wireless networks are deployed while supporting legacy infrastructures.

Despite RAT heterogeneity, the service model pursued under next-generation wireless networks is intended to facilitate the deployment of applications and services independent of the underlying RAT. Hence, it is expected that mobile users could eventually enjoy truly seamless mobility and ubiquitous service access in an always best connected mode, employing the most efficient combination of available access systems at any time and anywhere. In this con- text an appropriate interworking of different wireless access systems is crucial to meet mobile users' expectations while making possible the coexistence of diverse RATs.

The development of interworking solutions for heterogeneous wireless networks has spurred a considerable amount of research in this topic, especially in the context of IEEE 802.11 wireless local area networks (WLANs) and cellular network integration. Interworking is linked to many technical challenges such as the development of enhanced network architectures $[1,2]$, new mechanisms and protocols for seamless handover [3], and advanced management functionality for the joint exploitation of heterogeneous wireless networks [4, 5]. Accordingly, interworking aspects are receiving a lot of attention in standardization forums such as the Third Generation Partnership Project (3GPP), 3GPP2, Internet Engineering Task Force (IETF), WiMAX Forum, and IEEE 802 LAN/MAN Committee, the new IEEE 802.21 standard [6] for mediaindependent handover (MIH) being a clear exponent of such an effort.

While most published work is focused on particular interworking solutions for specific wireless technologies, this article establishes a comprehensive framework aimed at categorizing and analyzing interworking solutions. The proposed framework is based on the definition of a generic set of interworking levels along with a classification of the related key interworking mechanisms envisioned so far for heterogeneous wireless networks. The elaborated framework is then used to analyze from a common perspective some of the most relevant interworking solutions proposed for 3GPP, WLAN, and WiMAX networks. In particular, concerning the integration of WLAN and cellular networks, two interworking architectures specified by 3GPP, interworking WLAN (I-WLAN) [7] and generic access network (GAN) [8], are discussed. Next, in the context of coexisting mobile broadband access networks, interworking solutions for Mobile WiMAX and 3GPP Long Term Evolution/System Architecture Evolution (LTE/SAE) net- 
works are analyzed [9-11]. Finally, the MIH solution elaborated within IEEE 802.21 [6] is addressed.

The rest of this article is organized as follows. First, we describe a generic interworking scenario for heterogeneous wireless networks and bring up some major considerations about network architectures and multimode terminals. From such a basis, the proposed interworking framework is elaborated and the above mentioned interworking solutions are analyzed. The final section includes our main concluding remarks and discusses future trends.

\section{Wireless Heterogeneous INTERWORKING SCENARIO}

Figure 1 illustrates a generic interworking scenario for two coexisting wireless networks with partially overlapped coverage. It is assumed that the RAT used in each wireless network can be different, and terminals have multimode capabilities. Concerning service provisioning, a common set of services can be offered through both wireless networks (e.g., voice calls to/from public switched telephone networks), but there can also be some specific services only available when connected to a given wireless network (e.g., instant messaging and presence services).

Besides, as for network access rights, it is considered the most general view where two wireless networks could belong to different administrative domains (e.g., networks operated by different network service providers) but users can potentially be attached to either network (e.g., proper roaming agreements exist). Notice that a more particular case would be where the two networks form part of the same administrative domain (e.g., a mobile operator with Global System for Mobile Communications [GSM] and Universal Mobile Telecommunications System [UMTS] networks). In any case, in this article we denote as home network the network from which a user has obtained his/her credentials, and as visited network any other network to which the user can be connected.

In such a context, different interworking mechanisms would be needed to attend to the set of requirements imposed in terms of ubiquitous and seamless service access as well as of overall network resource optimization.

\section{Wireless Networks Characteristics}

Attending to current architectural trends in nextgeneration networks [12], wireless networks are mainly devoted to providing network connectivity services (i.e., bearer services) that may be characterized by a given quality of service (QoS) profile. Then, end user service provisioning is supported by means of specialized service platforms (e.g., IP multimedia subsystem [IMS]) that become accessible to the users via those bearer services.

Accordingly, Fig. 2 illustrates a generic wireless network architecture in terms of its main network nodes and protocol layer allocation. As shown in the figure, the wireless network provides network layer connectivity (e.g., IP connectivity) to external networks and service platforms via some type of network gateway (NG). In addi-

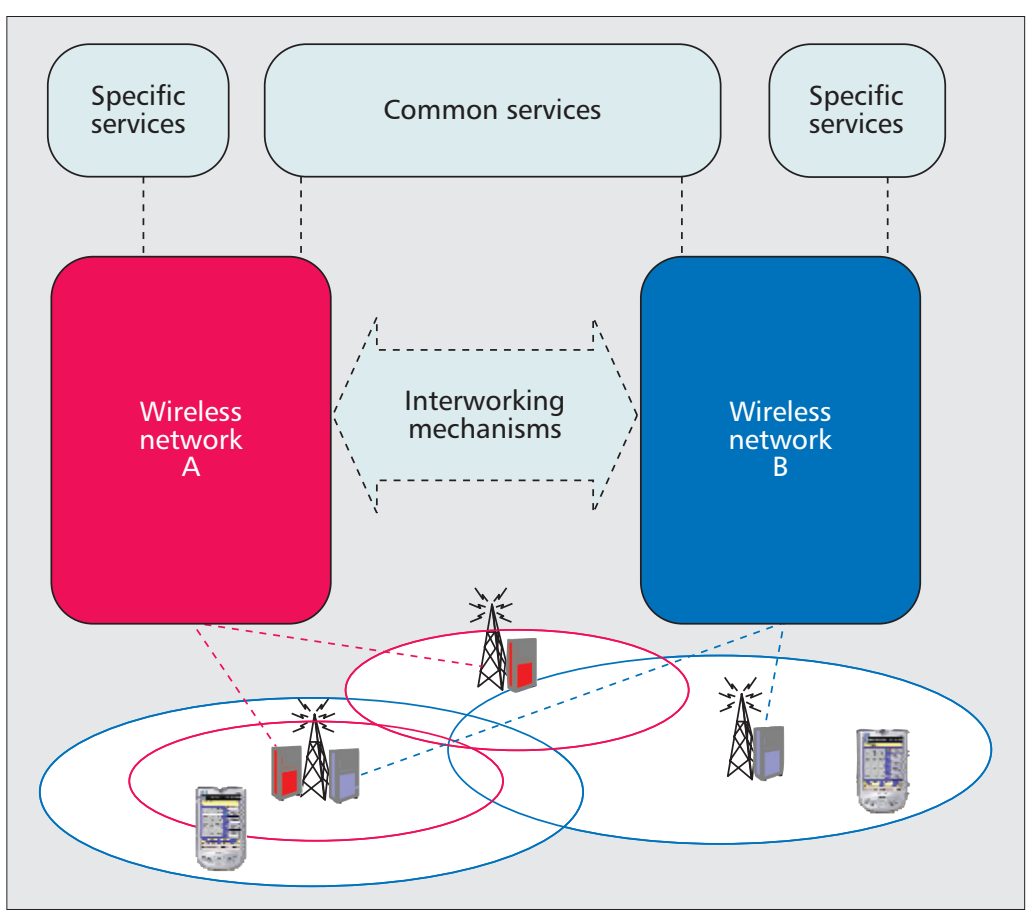

Figure 1. Generic interworking scenario for heterogeneous wireless networks.

tion, this NG can allocate mechanisms to dynamically acquire operator policies related to QoS and accounting, and enforce them on a packetby-packet basis for each mobile user. On the other side, a RAT-specific radio link protocol stack would be used in the air interface. This radio protocol stack can be entirely allocated in base stations (BSs) or distributed in a hierarchical manner between BSs and some type of radio controllers. The radio link protocol stack comprises physical, medium access control, and radio link control layers. Through this radio protocol stack, data transfer in the radio interface can be managed, attending to each mobile user's specific needs while simultaneously pursuing an efficient usage of radio resources by means of appropriate radio resource management (RRM) mechanisms. Hence, BSs and NGs constitute the two key elements within the data plane functions (i.e., those functions that are executed directly on the flow of data packets). Additionally, the data plane between BSs and NGs can also comprise mobility anchoring functions in charge of receiving data destined for a given mobile and redirecting the data (usually through tunneling) to the mobile's serving BS.

The management of the overall connectivity service is achieved by a network control plane. Unlike the data plane, the control plane functions are those that do not directly operate on the data flow. This network control plane would be in charge of handling mechanisms such as network access control (e.g., authentication and authorization), accounting and charging functions, mobility management (e.g., location and paging), security management, and session management. For the sake of brevity, all of the above mentioned control plane mechanisms are referred to as wireless network control (WNC) mechanisms throughout this article. Thus, as 


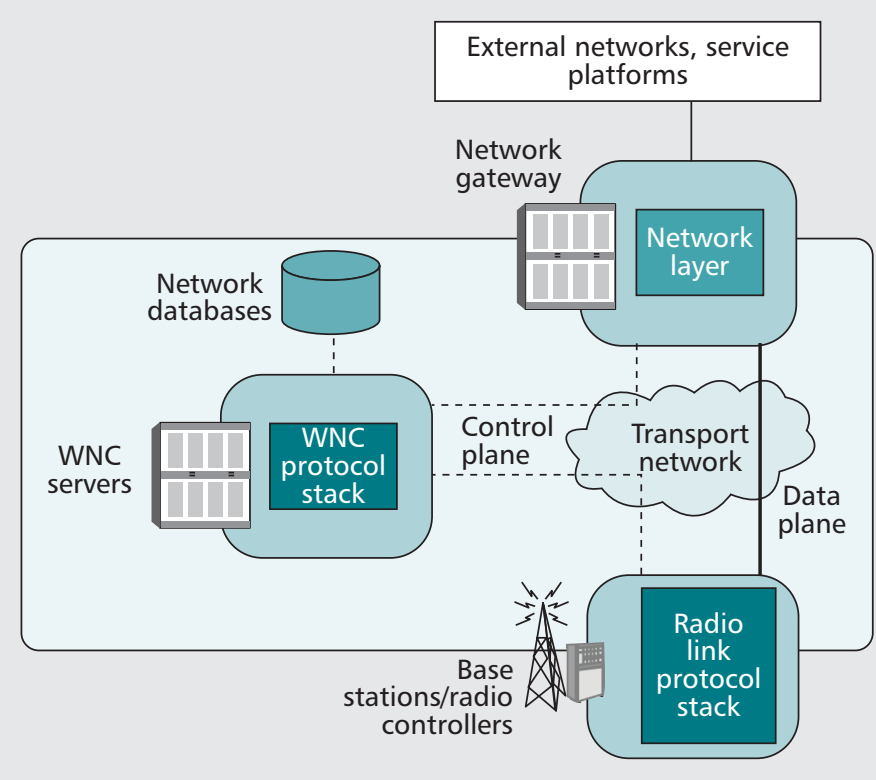

Wireless access network

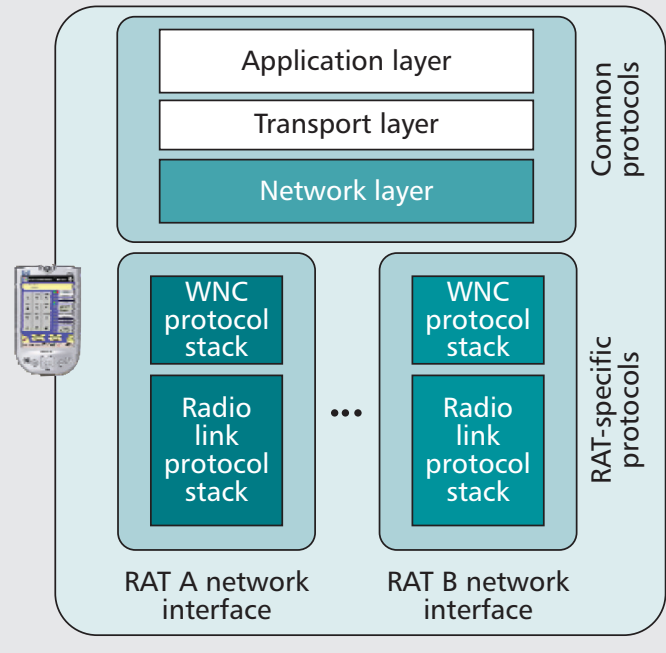

Multimode terminal

Figure 2. Generic architecture for a wireless access network and protocol stack of a multimode terminal.

shown in Fig. 2, a set of WNC servers, along with some network databases (e.g., subscriber profile databases), are assumed to allocate all these functions.

Finally, general-purpose packet-switched networks would constitute the backbone transport network that interconnects the different network nodes.

\section{Multimode Terminal Characteristics}

Focusing now on the key terminal characteristics, a simplified protocol stack of a multimode terminal is depicted in Fig. 2. This protocol stack consists of RAT-specific protocols for the lower layers (i.e., physical and link layers) and a common set of protocols for the higher layers (i.e., network, transport, and application layers). Concerning the RAT-specific protocols, they would comprise the correspondent radio link protocol stack to handle data transfer in the air interface along with the protocols used for WNC-related functionality in each wireless network. As to the common protocol layers, the network layer (e.g., IP) has a fundamental role in the interworking model since it provides a uniform substrate over which transport (e.g., Transmission Control Protocol [TCP] and User Datagram Protocol [UDP]) and application protocols (e.g., Session Initiation Protocol [SIP]) can efficiently run independent of the used access technologies.

In addition to protocol stack considerations, whether the terminal is able to transmit and receive simultaneously on both radio links (dual-radio operation) or only on one at a time (single-radio operation) has important implications on required interworking mechanisms, as discussed later.

\section{INTERWORKING LEVELS}

Bearing in mind all the above considerations, several interworking levels can be envisioned with a different range of interworking require- ments. The definition of interworking levels can be conducted attending to, say, network architecture aspects or the level of support for specific service and operational capabilities. In this work a definition exclusively based on the level of service integration among networks is considered because of its independence from underlying network technologies and architectures. Hence, in a general case, four interworking levels are distinguished.

\section{LeVel A: Visited Network Service Access}

This level of interworking would allow a user to get access to a set of services available in a visited network while relying on his/her home network credentials. As well, the user could be charged for service usage in the visited network through its own home network billing system. An example could be the case of a cellular subscriber, equipped with a laptop with both cellular and WLAN network interfaces, able to log into a public WLAN hotspot using its cellular smart card credentials and get high-speed Internet access from the hotspot service provider.

\section{LEVEL B: INTERSYSTEM SERVICE ACCESS}

In this level, users connected through a visited network would also be able to get access to specific services located in his/her home network. Hence, coming back to the example given in level A, the cellular subscriber using a cellular/WLAN laptop would also enjoy his/her cellular IMS services while attached to the public WLAN hotspot. Neither level A nor level B would support service continuity when the user moves between networks.

\section{LeVEl C: INTERSYSTEM SERVICE CONTINUITY}

This level extends the previous ones so that the user is not required to re-establish active session(s) when moving between networks. How- 


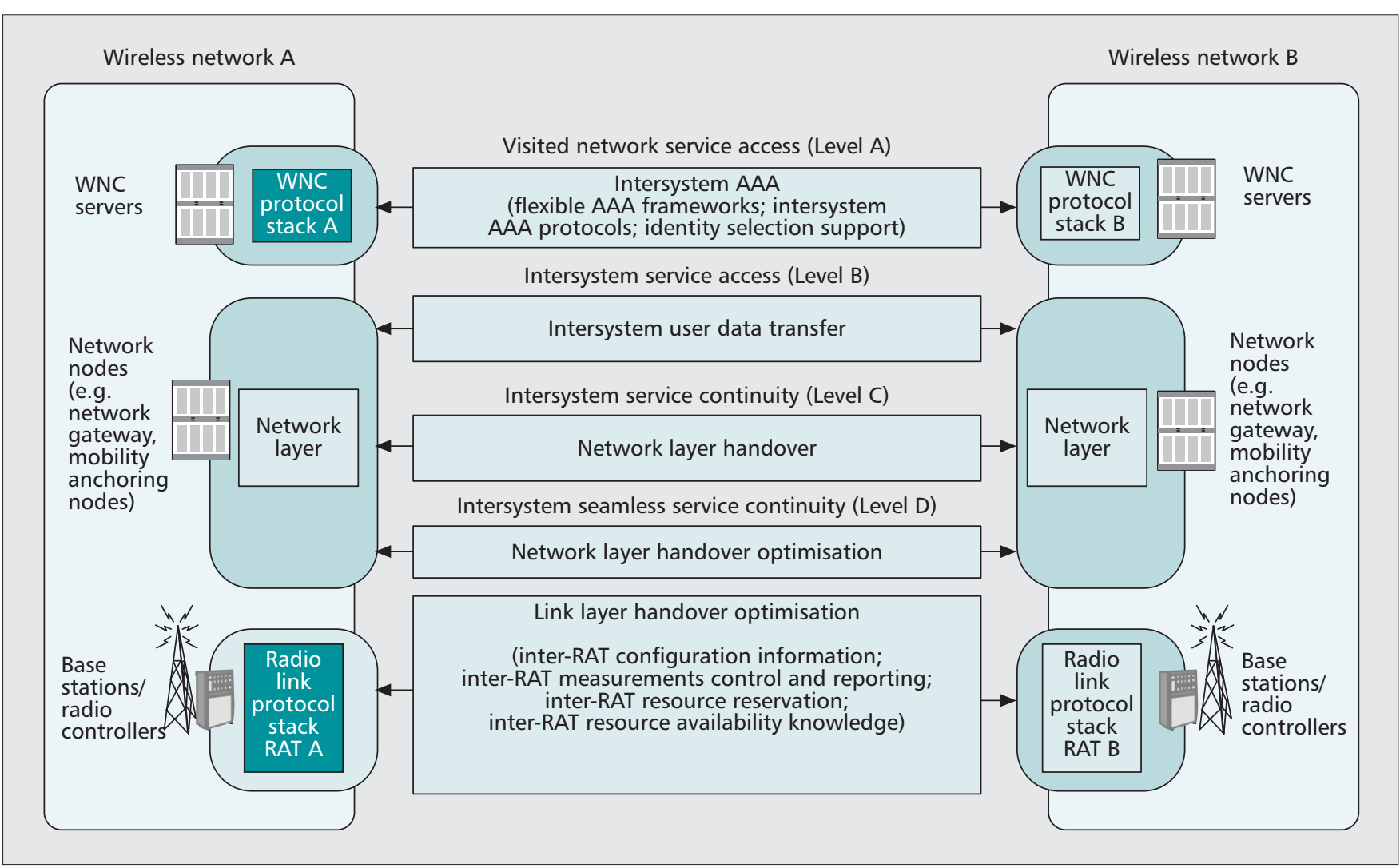

Figure 3. Interworking levels and related interworking mechanisms.

ever, a temporary QoS degradation can be tolerated during the transition time. As an example, if the cellular user equipped with the cellular/WLAN laptop begins to download a large file when attached to the public WLAN hotspot and the user moves so that WLAN coverage is lost, the downloading service will continue through the cellular network without user intervention even though a short transfer interruption might be observed during the network change.

\section{Level D: Intersystem Seamless SERVICE CONTINUITY}

This level is aimed to satisfy service requirements also during mobility (i.e., to offer a seamless mobility experience). Seamless service continuity can be achieved by enabling mobile terminals to conduct seamless handovers across diverse access networks. A seamless handover is commonly related to the achievement of low handover latencies (e.g., less than $300 \mathrm{~ms}$ could be required for real-time services in intertechnology handovers) so that this interworking level is the one that imposes the hardest requirements on the interworking mechanisms. As an example, focusing again on the dual-mode cellular/WLAN user, a voice over IP (VoIP) call established when attached to the WLAN hotspot should be seamlessly handed over to the cellular network. Finally, it is worth noting here that seamless service continuity is dependent not only on interworking mechanisms but also on the consistency of QoS characteristics provided by involved networks.

\section{INTERWORKING MECHANISMS}

Attending to the four interworking levels identified, hereafter we provide a classification of those interworking mechanisms that constitute the basic enablers/building blocks in each level. Figure 3 illustrates the addressed interworking levels and mechanisms, and relates them to the main network nodes and protocol layers within wireless networks previously illustrated in Fig. 2.

\section{LEVEL A: INTERSYSTEM AAA}

Mechanisms included here aim at extending authorization, authentication, and accounting (AAA) functions among wireless networks, allowing users to perform authentication and authorization processes in a visited network attending to security suites and subscription profiles provided by their home networks. As well, the deployment of one bill solutions advocates for the existence of mechanisms to transfer accounting and charging data between wireless networks. All these functionalities are basically achieved by:

- Adoption of flexible AAA frameworks able to support multiple authentication methods (e.g. the Extensible Authentication Protocol [EAP] defined in IETF RFC 3748 provides support for the reliable transport of different authentication protocols).

- Deployment of additional functionality such as AAA proxy/relay functions and related signaling interfaces between networks (e.g., the Diameter protocol defined in RFC 3588 provides the minimum requirements for an AAA protocol between networks). 
When focusing on

service continuity of

common services,

service continuity can

also be provided by

the service itself

(e.g., application-

based mobility

relying on SIP as

considered in [14])

without the need for

network layer han-

dover solutions.
- Enhanced network discovery mechanisms for identity selection so that mobile terminals could know in advance whether their home network's credentials are valid for AAA control in a visited network. Notice that nowadays, wireless networks do not provide such information over the air interface, and the typical approach is to have mobile terminals preconfigured with a list of allowed access networks.

\section{LEVEL B: INTERSYSTEM USER DATA TRANSFER}

These mechanisms enable the transfer of user data between networks in order to give access to specific services provided in a network other than the serving one. A common approach to enforce user data transfer between networks relies on tunneling protocols such as the Layer 2 Tunneling Protocol (L2TP) defined in RFC 2661 or the IPsec tunnel mode defined in RFC 2401. Tunnels may be established either directly between mobile terminals and remote NGs or may require additional dedicated network nodes. As an example, 3GPP specifications for WLAN access to 3 GPP packet-switched services [7] mandates the support of the IPsec Encapsulating Security Payload (ESP) protocol described in RFC 4303 for intersystem user data transfer.

\section{Level C: Network Layer Handover}

These mechanisms are required when service continuity between wireless networks relies on the maintenance of a permanent mobile terminal IP address. In this regard, Mobile IP (MIP) described in RFC 3344 was the initial proposed standard to serve the needs of globally mobile users who wish to connect to the Internet and maintain connectivity as they move from one network to another. MIP is a network layer mobility solution that covers both handover and location management aspects. MIP is based on a redirection approach achieved by a home agent (HA) functionality that maintains a binding between the global IP address assigned to the terminal (i.e., home address [HoA]) and the provisional IP address (i.e., care-of address [CoA]) allocated temporarily within the serving wireless network. Within the MIP solution, the mobile terminal itself is in charge of updating the address binding of its $\mathrm{HA}$ as the CoA changes (i.e., host-based mobility). On such a basis, several IP mobility protocols have been proposed over the past several years to complement or enhance MIP over IPv4 networks (e.g., reverse tunneling in RFC 3024) as well as IPv6 networks (e.g., MIPv6 described in RFC 3775 and Hierarchical MIPv6 for localized mobility described in RFC 5380). More recently, network-based IP mobility solutions where the terminal is not directly involved in managing IP mobility (e.g., Proxy MIPv6 defined in RFC 5213) are also being introduced in wireless networks [13]. As an example, the 3GPP LTE/SAE network allocates HA functionality for MIP-based mobility anchoring (either host-based or network-based) between LTE/SAE and other non-3GPP access networks [10]. It is important to mention here that, when focusing on service continuity of common services (i.e., those available in both home and visited networks as illustrated in Fig. 1), ser- vice continuity can also be provided by the service itself (e.g., application-based mobility relying on SIP as considered in [14]) without the need for network layer handover solutions.

\section{LeVel D: Network Layer Handover Optimization}

While simple network or application layer handover solutions may suffice for intersystem service continuity, they may not be able to satisfy the requirements for seamless mobility. In particular, during a handover, latencies related to radio link layers (e.g., new radio link establishment) and network layer operation (e.g., movement detection, new IP address configuration, and binding updates) can turn into a period during which the terminal is unable to send or receive packets. In this respect, several optimization mechanisms have been proposed to reduce the handover latency due to network layer operations. As an example, RFC 4068 defines fast handover extensions for MIPv6 so that terminals can acquire a new valid CoA before a handover occurs, and where tunneling between the old and new CoAs reduces the binding update latency. Besides, when handover takes place between networks in different administrative domains, pre-authentication schemes such as the one proposed in [15] can help reduce non-negligible authentication and authorization delays. Also, context transfer mechanisms (e.g., Context Transfer Protocol [CXTP] defined in RFC 4067) can be used to preconfigure different network (and also link) layer parameters in the target network and so avoid re-initiation of some signaling to and from the terminal. Finally, network discovery mechanisms (see RFC 5113 for a detailed discussion) also have a crucial role in handover optimization since they can convey specific information needed for optimized mobility.

\section{Level D: Link Layer Handover Optimization}

Radio link layer operations could also introduce delays in the handover process. Hence, setting up the new radio link could take several steps (e.g., scanning, authentication, and association in 802.11) that handover optimization mechanisms should have to either bypass or minimize the latency of while connected to the previous link, especially for the single-radio operation case. At the same time, handover optimization mechanisms can allow for more efficient radio resource usage (e.g., network-controlled interRAT mobility). Hence, among the main aspects covered by such mechanisms we have:

- Provision of inter-RAT configuration information about neighboring BSs to enhance the radio scanning process and guide network selection decisions.

- Inter-RAT measurements control and reporting to improve handover initiation.

- Inter-RAT resource reservation. This could imply the preconfiguration of some radio link contexts (e.g., QoS contexts) in the target RAT.

- Inter-RAT resource availability knowledge (e.g., check or reporting procedures) in order to enhance handover decisions. 


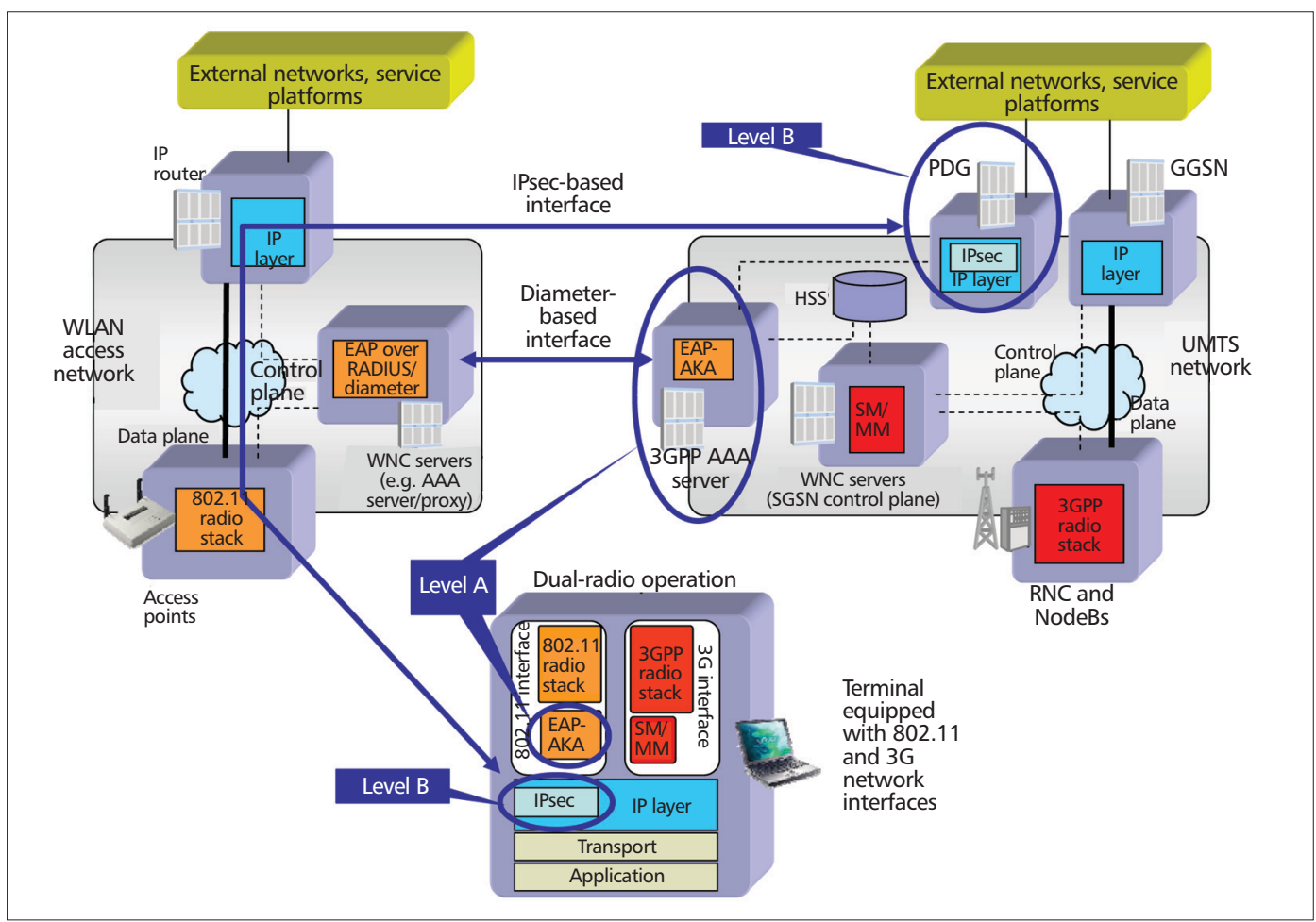

Regarding Level B,

IPsec ESP is used to

provide secure

tunnels between

terminals connected

to the WLAN and a

new node named

Packet Data

Gateway (PDG)

within the 3GPP

network. The PDG

basically behaves

as a GGSN for

WLAN users.

Figure 4. I-WLAN interworking model.

As an example, inter-RAT handover mechanisms specified between 3GPP GSM and UMTS networks cover most of the aforementioned aspects.

\section{DISCUSSION ON RELEVANT INTERWORKING APPROACHES}

According to previous interworking levels and related mechanisms, in this section we analyze some of the most relevant proposed solutions.

\section{I-WLAN}

I-WLAN architecture [7], commonly referred to as loose coupling interworking, is targeted to cover interworking levels A and B for packet services (i.e., General Packet Radio Service [GPRS]). Following the basic architecture representation for a generic wireless network introduced in Fig. 2, Fig. 4 illustrates both the main network elements within 3GPP UMTS and WLAN networks and the new main network functions (marked within circles) and interfaces (highlighted in italics) added for I-WLAN interworking support.

As to the 3GPP UMTS network, network gateway functions are provided by the gateway GPRS support node (GGSN); WNC functions such as mobility management (MM) and session management (SM) are mainly handled within the control plane of the serving GSN (SGSN) and with the support of a home subscriber server (HSS) database; and UMTS radio link protocol stack and related functions (e.g., RRM) are distributed among NodeBs (i.e., naming convention for UMTS BSs) and radio network controllers (RNC). As for the WLAN access network, the IEEE 802.11 protocol stack (e.g., MAC and physical layer) is entirely handled by access points (APs); WNC functions such as access control can be supported by means of AAA servers using IETF protocols (e.g., RADIUS protocol between APs and AAA servers for EAP/IEEE 802.1X access control); and generic routers can provide NG functions to external networks or service platforms.

Thus, interworking level $\mathrm{A}$ is achieved by the allocation of a 3GPP AAA server in the 3GPP network and AAA proxy functions within the WLAN network. The interface between them is based on the Diameter protocol, which can be used for the transfer of, say, EAP messages for authentication and authorization. In this respect, specific EAP extensions for 802.1X access control have been defined to allow authentication based on UMTS credentials (i.e., EAP-AKA defined in RFC 4187). As well, the list of available interworking UMTS networks can be provided through the WLAN connection by means of the network discovery mechanism defined in RFC 4284.

Regarding level B, IPsec ESP is used to provide secure tunnels between terminals connected to the WLAN and a new node named packet data gateway (PDG) within the 3GPP network. The PDG basically behaves as a GGSN for WLAN users.

From the terminal side, the I-WLAN model basically requires a conventional $3 \mathrm{G}$ network interface, a 802.11 network interface supporting EAP/802.1X authentication (e.g., Wireless Protected Access [WPA] certification) and support for IPsec within the IP protocol stack.

\section{Generic AcCess Network}

The GAN [8], also referred to as tight coupling interworking, provides a solution to extend cellular circuit and packet services over IP broadband 
The initial interworking solution considered within WiMAX Forum and 3 GPP is based on the I-WLAN architecture by 3GPP. Hence, such a solution is already available for the first release of the WiMAX architecture (WiMAX NWG Release 1) and covers interworking Levels A and B.

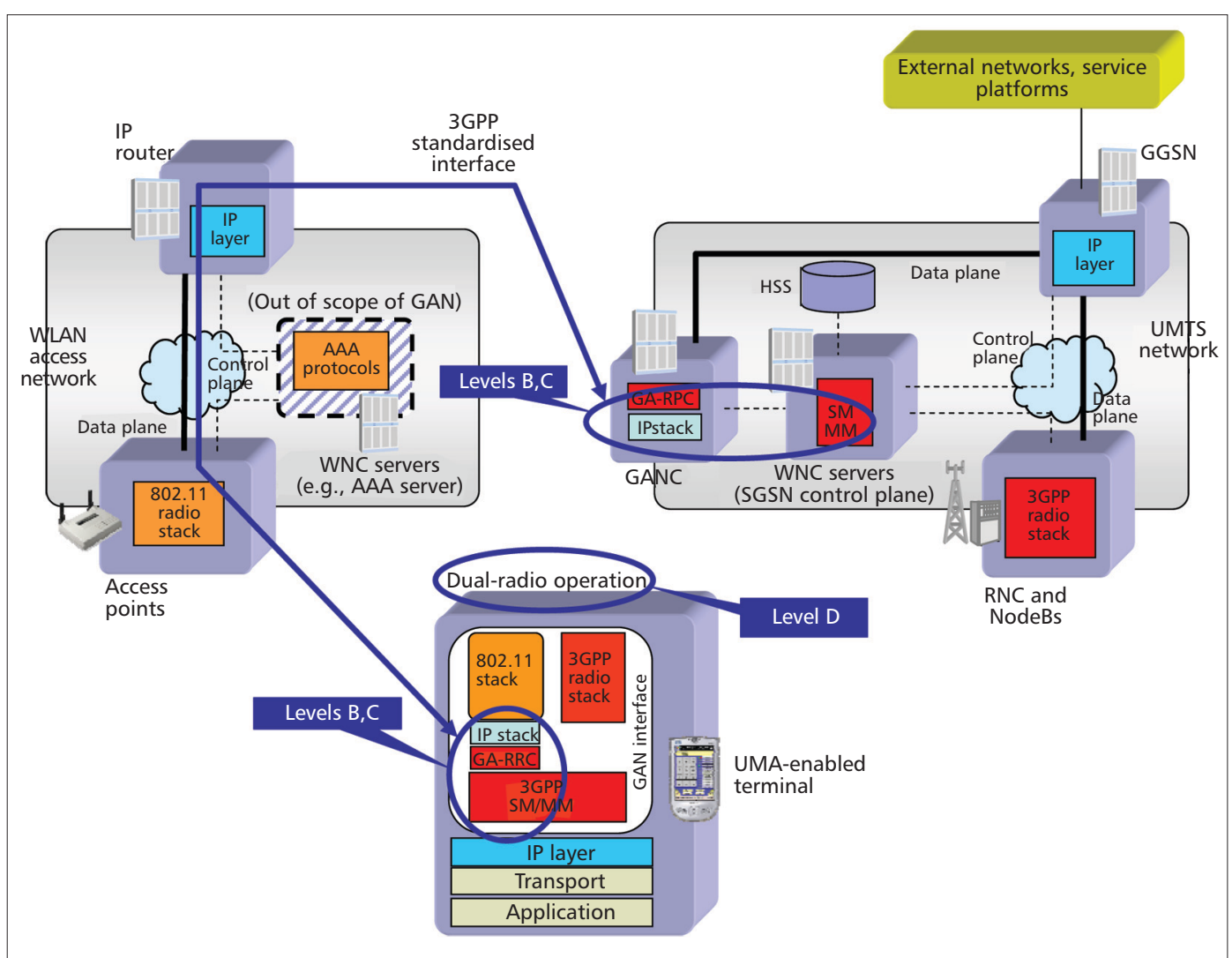

Figure 5. GAN interworking model.

access networks. Hence, the GAN model is not restricted to 802.11 access networks, yet deploying GAN over 802.11 is the most common approach. Moreover, dual-mode cellular/802.11 terminals compliant with GAN specifications are more widely known as unlicensed mobile access (UMA)-enabled terminals.

The GAN model provides interworking levels B, C, and D. Figure 5 illustrates the main aspects of the GAN interworking approach within the same UMTS/WLAN scenario previously described for I-WLAN. The three key elements of the GAN model, as shown in Fig. 5, are a new network element named GAN controller (GANC) located within the 3GPP network, a UMA-enabled terminal, and a new interface between both elements specified by 3GPP.

The GANC serves much like an RNC, reusing legacy $3 \mathrm{GPP}$ interfaces towards the core network. Regarding the UMA-enabled terminal, it is a 3 GPP terminal with embedded 802.11 communications. Hence, the interface defined between GANC and UMA-enabled terminals comprises new protocols with functions similar to the UMTS radio resource control (RRC) protocol (e.g., Generic Access RRC [GA-RRC]) along with legacy 3 GPP control plane protocols (e.g., SM and MM). The transfer of all the information between the terminal and the GANC through the WLAN network uses IPsec tunneling mechanisms.

On this basis, interworking level B is provided by the fact that GANC actually constitutes a gateway toward 3GPP services. Also, level C is built up on legacy 3GPP handover procedures so that established data sessions or calls could be transferred between the GANC, acting as an RNC from the network side, and the corresponding RNCs within the UMTS network without service disconnection. Concerning level D, handover latencies similar to those between UMTS cells can be achieved by the fact that UMA-enabled terminals support dual-radio operation. Hence, a UMA-enabled terminal can connect to the GANC via a WLAN while maintaining the UMTS network connection so that the aforementioned interworking mechanisms for network or link layer handover optimization are not necessary. On the contrary, level A is out of the scope of the GAN specifications since the GAN model only requires IP connectivity from the WLAN access network and does not deal with 802.11-specific issues such as access control.

\section{WIMAX AND 3GPP NETWORKS INTERWORKING}

The initial interworking solution considered within the WiMAX Forum and 3GPP is based on the IWLAN architecture by 3GPP. Hence, such a solution is already available for the first release of the WiMAX architecture (WiMAX NWG Release 1 [9]), and covers interworking levels A and B. Then, in the context of 3GPP LTE/SAE networks, architecture enhancements are being specified for providing IP connectivity using non-3GPP accesses [10]. In particular, support for MIP and PMIPbased mobility is being introduced to achieve level C. As well, functionality intended to provide network discovery and selection assistance data is being specified (i.e., access network discovery and selection function [ANDSF]).

Besides, optimized interworking solutions between WiMAX networks and 3GPP LTE/SAE 


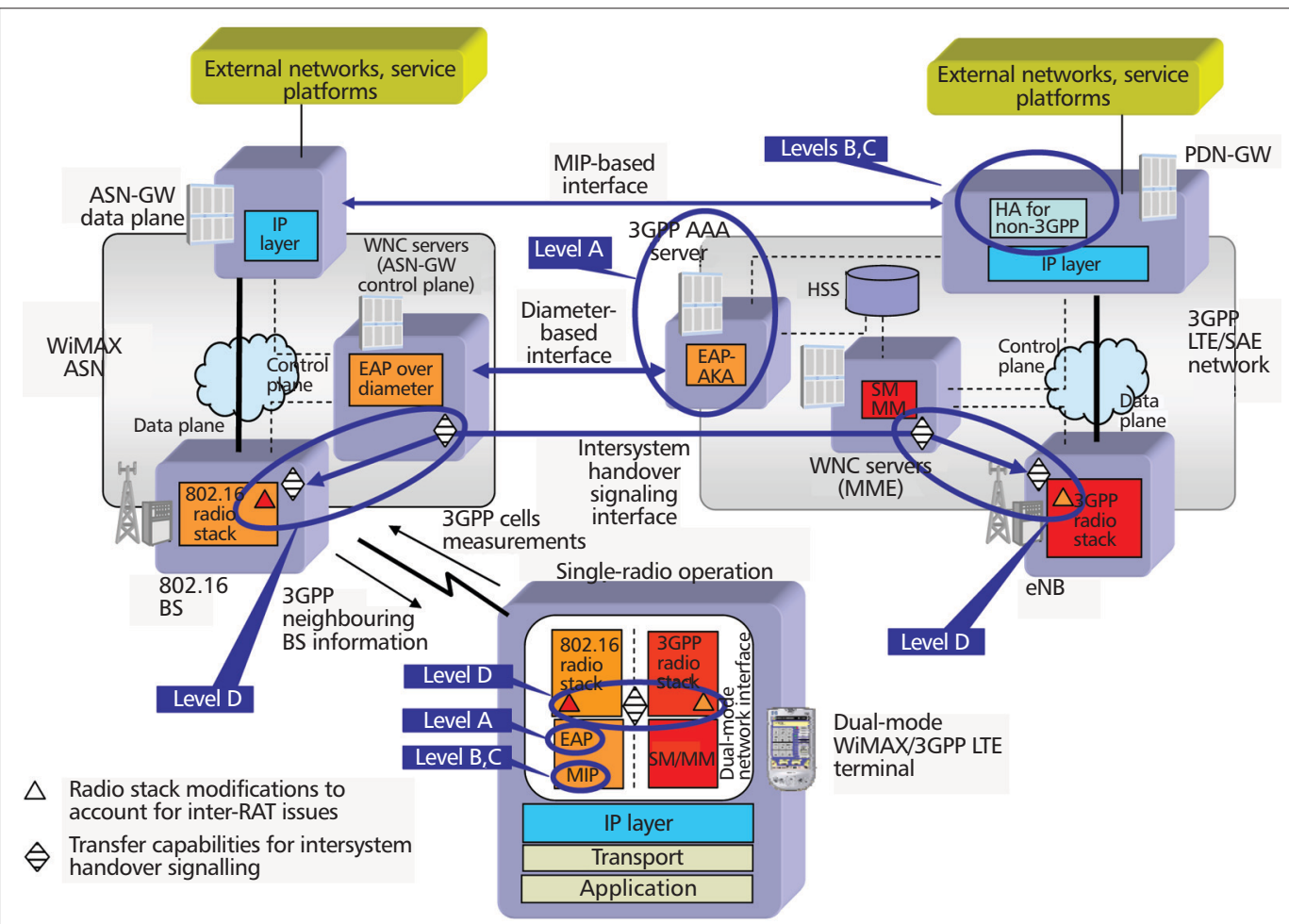

It is worth noting that for dual-radio operation, previous mechanisms would not be necessary. Nevertheless, dual-radio operation may not be feasible due to, say, radio frequency coexistence issues when radio frequencies used in the two RATs are close to each other.

Figure 6. WiMAX and 3GPP LTE/SAE interworking model.

networks are also under consideration to achieve interworking level D [11]. In this regard, an approach targeted to cover interworking levels A, B, C, and D is illustrated in Fig. 6 and discussed next for a 3GPP LTE/SAE network coexisting with a WiMAX access service network (ASN). As shown in Fig. 6, regarding the 3GPP LTE/SAE network, radio link layer protocols and functions are now entirely allocated in an enhanced NodeB (eNB), and an evolved packet core (EPC) handles WNC functions via so-called MME servers and provides NG functionalities through PDN gateways (PDN-GWs). As for the WiMAX ASN, the IEEE 802.16 e radio protocol stack is also entirely allocated to BSs, and a separate network node named ASN gateway (ASN-GW) could hold both data plane anchoring functionalities (e.g., for mobility anchoring within WiMAX ASN) and control plane WNC functions (e.g., authentication based on an EAP framework).

According to this interworking solution, interworking level $\mathrm{A}$ is achieved as in I-WLAN solution by means of AAA proxy functions in the ASN-GW, a 3GPP AAA server located in the 3GPP LTE/SAE network, and the use of specific EAP authentication mechanisms. Then levels B and $\mathrm{C}$ are built up on MIP-based mobility solutions already supported within the WiMAX ASN (e.g., the ASN-GW data plane can allocate foreign agent [FA] functions for MIPv4 or access router [AR] functions for MIPv6) and on adding data plane anchoring functions within the $3 \mathrm{GPP}$ LTE/SAE network (e.g., HA functions for MIPv4 or MIPv6 are allocated in the PDN-GW). The MIP client can be located in the terminals, as shown in Fig. 6, or in the ASN-GW if PMIP solutions are used instead between the ASN-GW data plane and the 3GPP PDN-GW.
Concerning interworking level D, intersystem handover optimization mechanisms are discussed in [11] to provide seamless mobility for single-radio operation terminals, a condition that imposes the hardest requirements on the interworking solution. In this respect, as illustrated in Fig. 6, both WiMAX and $3 \mathrm{GPP}$ LTE/SAE radio link protocol stacks would need to be modified to add support for inter-RAT measurement control and reporting along with the delivery of inter-RAT BS information. Besides, support for resource reservation, as well as pre-registration (i.e., covering aspects such as access control, context transfers and default bearer service establishment), is envisaged by means of tunneling legacy signaling messages between terminals and BSs or WNC servers in the target network while being attached to the serving network. Hence, as example, a terminal connected to an eNB can initiate a handover to WiMAX by tunneling a 802.16e Handover Request message through the 3GPP LTE/SAE network towards the target WiMAX BS. Transparent transfer of intersystem handover signaling messages requires each radio protocol stack to provide transport functionality for signaling messages of a different technology. Moreover, transparent signaling transfer between networks requires the deployment of an interface among MME and ASN-GW Control Plane as shown in Fig. 6 (e.g., S101 interface in [11]) for intersystem handover signaling. Finally, it is worth noting that for dual-radio operation, previous mechanisms would not be necessary. Nevertheless, dual-radio operation may not be feasible due to e.g., radio frequency coexistence issues when radio frequencies used in the two RATs are close to each other. 
IEEE 802.21

solution is expected

to constitute a cor-

nerstone within IEEE

802's proposal for

IMT-Advanced, where

enhanced IEEE

802.16 and IEEE

802.11 radio

technologies will be

integrated under the

interworking model

offered by 802.21 .
IEEE 802.21 INTERWORKING

The standard IEEE 802.21 "Media-Independent Handover (MIH) Services" [6] is mainly conceived as an enabler of interworking level D between heterogeneous link-layer technologies (e.g., IEEE 802 and cellular networks) and across IP subnet boundaries. The standard defines a new link layer functionality (i.e., MIH Function [MIHF]) to be added within terminals and networks together with the protocol required for message exchanging between them. This MIHF entity is allowed to have some control on link layer behavior (e.g., link actions such as power-up and link configuration) and can collect link information (e.g., link status polling or event-triggered information). To that end, radio link layers have to add support for a media specific interface with the MIHF function. On the other hand, the MIHF entity provides a media independent interface to upper layers of the protocol stack (denoted as MIH users in 802.21 standard's notation) and offers a set of generic services classified as event, command and information services [3]. Through these services, upper layers can control lower layer's behavior (e.g., remote/local link actions and handover commands) and acquire relevant information for more efficient handover decisions (e.g., local/remote link status and information about different available networks and their services). The 802.21 service model offers a flexible framework to facilitate different handover approaches. Hence, while served by a given wireless network, the MIHF entity of the mobile terminal could interact with a MIHF entity in the serving network in order to retrieve inter-RAT network information and initiate an inter-technology handover by indicating a preferred list of candidate access networks. As well, handover could also be initiated from the network side. In both cases, 802.21 signaling enables intersystem radio resource availability check and resource preparation (e.g., intersystem resource reservation) between involved networks and provides the terminal with the required configuration of the reserved resources at the target network. It's worth noting that the scope of 802.21 is limited to handover initiation and preparation phases, while the execution phase is not covered (e.g., mobility handling in upper layers is still required for service continuity between networks).

The adoption of the 802.21 solution in a WiMAX-3GPP LTE/SAE interworking scenario such as the one described in Fig. 6, would require the allocation of functional MIHF entities in the terminal and within both wireless networks along with the correspondent transport capabilities to exchange MIH protocol messages. In this sense, MIH signaling to/from terminals could be transferred by using specific mechanisms introduced in the radio link layers (IEEE 802.16g extension adds such a support but 3GPP does not consider it yet). Another possibility would be the transfer of MIH signaling op top of the IP bearer service provided by the network (e.g., IETF MIPSHOP working group is specifying support for sending 802.21 messages over IP networks).

\section{CONCLUDING REMARKS AND FUTURE TRENDS}

This article has elaborated a comprehensive framework to analyze and categorize interworking solutions in heterogeneous wireless networks. Over such a basis, some of the most relevant interworking solutions being considered in different standardization bodies have been discussed.

As to WLAN and cellular interworking solutions, I-WLAN has been noted to constitute a simple but versatile solution to extend cellular packet services access. I-WLAN requires little changes in terminals and networks, though it is not able to provide seamless mobility and network-controlled mobility. Currently, I-WLAN interworking architecture is being extended to handle mobility between I-WLAN and 2G/3G by means of adding support for MIP-based mobility, thus converging to the type of solutions being considered within LTE/SAE for interworking with non-3GPP access networks. On the other hand, 3GPP GAN solution requiring dual-radio operation UMA-enabled terminals was firstly developed for $2 \mathrm{G}$, then extended to $3 \mathrm{G}$ but no extension is envisioned so far within LTE/SAE, where PDN-GW intersystem mobility anchoring with MIP and PMIP-based solutions are favored. Nevertheless, UMA-terminals are a reality today as some operators are embracing such a technology both in business and in the home 802.11/cellular access.

Concerning possible solutions to achieve optimized seamless handover with single-radio terminals between LTE/SAE and WiMAX, two main approaches have been discussed. While 3GPP envisions a tailored solution, IEEE 802.21 efforts are trying to push for a generic solution for inter-technology handover. Nevertheless, the adoption of a generic solution such as IEEE 802.21 in 3 GPP networks is not as straightforward as within IEEE-based technologies, which already share a common architectural framework. In this regard, the 802.21 standard is becoming a central piece of an IEEE 802 wide initiative so that, in addition to defining the new 802.21 standard itself, IEEE is making changes to existing access technologies specifications (e.g., WLAN, WiMAX) to support 802.21 related handover functionality. Moreover, IEEE 802.21 solution is expected to constitute a cornerstone within IEEE 802's proposal for IMTAdvanced [3], where enhanced IEEE 802.16 and IEEE 802.11 radio technologies will be integrated under the interworking model offered by 802.21.

\section{ACKNOWLEDGMENTS}

This work has been partly funded by the Spanish Ministry of Science and Innovation under the TelMAX project, belonging to the Ingenio 2010 program. This work has also been partly supporrted by the Spanish Research Council and FEDER funds under a COGNOS grant (ref. TEC2007-60985. The authors are very thankful to the reviewers for their constructive comments. 


\section{REFERENCES}

[1] A.K. Salkintzis, C. Fors, and R. Pazhyannur, "WLAN-GPRS Integration for Next-Generation Mobile Data Networks," IEEE Wireless Commun., vol. 9, no. 5, Oct. 2002.

[2] A.K. Salkintzis, "Interworking Techniques and Architectures for WLAN/3G Integration toward 4G Mobile Data Networks," IEEE Wireless Commun., vol. 11, no. 3, June 2004.

[3] L Eastwood et al., "Mobility using IEEE 802.21 in a Heterogeneous IEEE 802.16/802.11-based, IMT-Advanced (4G) Network," IEEE Wireless Commun., vol. 15, no. 2, Apr. 2008.

[4] C. Skianis, G. Kormentzas, and K. Kontovasillis, "Interactions between Intelligent Multimodal Terminals and a Network Management System in a B3G Context," Wireless Commun. Mobile Comp., vol. 5 , no. 6, Sept. 2005.

[5] C. Skianis et al., "Efficiency Study of the Information Flow Mechanism Enabling Interworking of Heterogeneous Wireless Systems," J. Sys. Software, vol. 80, no. 10, Oct. 2007.

[6] IEEE Std 802.21, Draft D14.0, “IEEE Standard for Local and Metropolitan Area Networks: Media Independent Handover Services," Sept. 2008.

[7] 3GPP TS 23.234 v. 7.7.0, "3GPP System to Wireless Local Area Network (WLAN) Interworking; System Description (Release 7)," June 2008.

[8] 3GPP TS 43.318 v. 8.3.0, "Radio Access Network; Generic Access Network; Stage 2 (Release 7)," Aug. 2008.

[9] WiMAX Network Forum Architecture, "Stage 2, 3GPPWiMAX Interworking," Release 1, v. 1.2, Jan. 2008

[10] 3GPP TS 23.402 v. 8.2.0, "Architecture Enhancements for Non-3GPP Accesses (Release 8)," June 2008.

[11]. 3GPP TR 36.938 v. 8.0.0, "Improved Network Controlled Mobility between E-UTRAN and 3GPP2/Mobile WiMAX Radio Technologies," Mar. 2008.

[12] R. Agrawal and A. Bedekar, "Network Architectures for 4G: Cost Considerations," IEEE Commun. Mag., vol. 45, no. 12, Dec. 2007, pp. 76-81.
[13] K.-S. Kong et al., "Mobility Management for All-IP Mobile Networks: Mobile IPv6 vs. Proxy Mobile IPv6," IEEE Wireless Commun., vol. 15, no. 2, Apr. 2008, pp. 36-45.

[14] S. Salsano et al., "SIP-based Mobility Management in Next Generation Networks," IEEE Wireless Commun., vol. 15, no. 2, Apr. 2008, pp. 92-99.

[15] A. Dutta et al., "Media-independent Pre-authentication Supporting Secure Interdomain Handover Optimization," IEEE Wireless Commun., vol. 15, no. 2, Apr. 2008, pp. 55-64.

\section{BIOGRAPHIES}

RAMON FERRUS (ferrus@tsc.upc.edu) is an associate professor at Universitat Politècnica de Catalunya (UPC), Spain, since 2002. His actual research interest is focused on architectures, QoS mobility, and resource management in the context of heterogeneous IP-based mobile communications systems. He has participated in several research and technology transfer projects. He is co-author of more than 50 papers published in international journals/magazines and conference proceedings.

ORIOL SALLENT (sallent@tsc.upc.edu) is an associate professor at UPC. His research interests are in the field of radio resource and spectrum management for heterogeneous cognitive wireless networks, where he has published $100+$ papers in IEEE journals and conferences. He has participated in many research projects and consultancies funded by either public organizations or private companies.

RAMON AGUSTI (ramon@tsc.upc.edu) has been a full professor at UPC since 1987. For the last 20 years he has mainly been interested in mobile communications topics and has published about 200 papers in these areas. He has also participated in the COST and many European research programs, and managed many private and public funded projects. His actual research interests include radio networks, cognitive radio, radio resource management, and QoS. 